\title{
DISCURSOS REFLEXIVOS DE ESTUDIANTES INDÍGENAS EN UNIVERSIDADES PÚBLICAS DE CUÑO TRADICIONAL DE OAXACA, MÉXICO ${ }^{1}$
}

\author{
Gabriel Cruz ${ }^{2}$
}

\begin{abstract}
RESUMEN
En este artículo se exponen los resultados de una investigación acerca de discursos reflexivos de estudiantes indígenas y otros actores de dos universidades públicas de cuño tradicional del estado de Oaxaca, México. En una etapa en la que discursos y acciones impulsadas por el Estado mexicano han privilegiado el diseño y operación de instituciones interculturales, así como de programas o proyectos específicos en determinadas universidades, los diferentes actores perciben que el reconocimiento de la diversidad lingüística y cultural y el desarrollo de la educación intercultural se encuentran en una fase de instalación inaugural. Sobre la base de una revisión documental del tema, del análisis de narrativas autobiográficas y de entrevistas de estudiantes indígenas, académicos y administradores desde el enfoque de la reflexividad sociolingüistica se presentan algunas de las creencias y formatos respecto de la gestión académica y cultural, los principios éticos y valores de convivencia más significativos, así como de las regulaciones de cambio sociocultural señaladas por estos actores. Se puede concluir que existen esfuerzos para afrontar y remediar las desigualdades e inequidades que viven los estudiantes indígenas, sin embargo, los principios y filosofía de una "interculturalidad para todos" se encuentran distantes del desarrollo educativo intercultural que se pregona en el país.
\end{abstract}

Palabras clave: reflexividad sociolingüística, discursos reflexivos, estudiantes indígenas, universidades públicas, desarrollo educativo intercultural.

\section{REFLEXIVE DISCOURSES OF INDIGENOUS STUDENTS IN TRADITIONAL PUBLIC UNIVERSITIES OF OAXACA, MEXICO}

\begin{abstract}
This article presents the results of an investigation into reflexive discourses of indigenous students and other actors at two traditional public universities in the state of Oaxaca, Mexico. In a stage where discourse and actions promoted by the Mexican government have favored the design and operation of cross-cultural institutions as well as specific programs or projects in selected universities, the various actors understand that the recognition of linguistic and cultural diversity and the development of intercultural education are in a phase of initial development. Based on a documental review of the topic and an analysis of autobiographical narratives and interviews with indigenous students, academics and administrators, from the perspective of sociolinguistic reflexivity, the article presents participants' perceptions and beliefs about academic and cultural management, ethical principles and significant values of coexistence, and cultural strategies for change. The analysis indicates that although there are efforts to address and remedy the inequalities and inequities experienced by indigenous students, the principles and philosophy of "interculturality for all" are far from realized in the intercultural education currently underway in Mexico.
\end{abstract}

Keywords: reflexivity sociolinguistics, reflexive discourses, indigenous students, public universities, intercultural educational development.

1 Este artículo deriva de un trabajo de investigación colectiva realizado entre 2008 y 2014. Proyecto CONACYT 99274/2008 "Dominio lingüístico y académico de estudiantes bilingües indígenas en algunas universidades de México: habilidades comunicativas y académicas, reflexividad sociolingüística y políticas interculturales del lenguaje".

2 Universidad Pedagógica Nacional Unidad 201-Oaxaca, México. Contacto: GAELTH007@ gmail.com 


\section{Introducción}

En este texto se muestran los resultados de una investigación desarrollada en dos universidades públicas de modelo tradicional del estado de Oaxaca, México: la Universidad Pedagógica Nacional Unidad 201 (UPN 201) y la Universidad Autónoma Benito Juárez de Oaxaca (UABJO). Se plantea que los discursos reflexivos de estudiantes indígenas y otros actores constituyen una puerta de acceso a informaciones, formatos de uso público, valoraciones, declaraciones de intenciones y regulaciones de cambio sociocultural a favor de la lengua, la cultura y la educación intercultural, desde una perspectiva de investigación cualitativa denominada reflexividad sociolingüística (Muñoz, 1983, 2010a).

Este estudio forma parte de un campo emergente en el que discursos y acciones del Estado mexicano promueven el diseño y operación de instituciones interculturales, así como programas o proyectos específicos en determinadas universidades. No obstante, se demuestra que el reconocimiento de la diversidad lingüística y cultural así como el desarrollo de la educación intercultural se encuentran en una fase de desarrollo inicial.

Aunque en Latinoamérica existen estudios acerca de las políticas institucionales, los logros, retos y evaluaciones de programas específicos tanto en universidades interculturales como en universidades "convencionales" (Badillo, Casillas \& Ortiz, 2008; Didou y Remedi, 2006; Dietz, 2012; Mato 2008, 2009; Nivón, 2006; Romo y Hernández, 2005), las pesquisas en cuanto al acceso, financiamiento y matrícula escolar (Didou y Remedi, 2009), los avances en el desarrollo curricular (Velasco y Joblanska, 2010) y la operación de programas de tutorías (Badillo, 2008), son pocos los trabajos acerca de la reflexividad sociolingüística de estudiantes indígenas en las denominadas universidades convencionales (Muñoz, 2010b; Ortega, 2013).

Por lo tanto, el objetivo de este trabajo es acercarse a los discursos reflexivos de estudiantes indígenas, académicos y administradores para identificar los razonamientos o formatos 
asociados a la gestión académica y sociocultural, los valores y principios éticos de convivencia más significativos, así como a las declaraciones de intenciones o regulaciones de cambio sociocultural impulsadas por dichos actores.

Los resultados sugieren que los cambios a favor de la lengua, la cultura y la educación provendrán principalmente de las redes sociales de estudiantes y de los sectores donde estos actores se desenvuelven profesionalmente. Aunque se perciben esfuerzos institucionales para remediar las desigualdades e inequidades, los principios y filosofía de una "interculturalidad para todos" (Schemelkes, 2003) se encuentran distantes de los cambios que se pregonan.

A partir de la revisión de información documental disponible sobre estudiantes indígenas en la educación superior y del análisis de narrativas autobiográficas y entrevistas, se expone una selección de temas y tópicos, así como un esquema del funcionamiento reflexivo de los niveles del desarrollo educativo intercultural (Muñoz, 1998, 2002), que el diseño de políticas educativas debiera considerar.

\section{La educación superior indígena en México}

La educación superior ofertada en México y otros países en Latinoamérica, hasta el momento, ha privilegiado una política monocultural y monolingüe de la universidad mexicana, promoviendo la asimilación e inequidad lingüística y cultural en la educación superior. El multilingüismo y el plurilingüismo son asuntos marginales y aislados en el proyecto de la educación superior, y los procesos de enseñanza y aprendizaje para los estudiantes etnolingüísticamente diferenciados se ofrecen generalmente en programas de formación lingüística y antropológica, sin obviar el papel central de los modelos pedagógicos y psicolingüísticos en el diseño de los mismos.

La comunidad universitaria refleja el ethos diglósico de jerarquías socioculturales y la discriminación que impera en la sociedad mexicana. Además, el desconocimiento de la diversidad lingüística y cultural y de la comunicación intercultural es parte constitutiva del universo gnoseológico de la mayoría de los sectores 
universitarios y de la sociedad en general. Puede ser que la aprobación retórica y pasiva de la diversidad lingüística y cultural, dentro y fuera de las aulas por parte de los estudiantes indígenas, sea el hecho más visible.

Así mismo, las posibles barreras cognitivas y comunicativas atribuibles a factores fenotípicos y étnicos traen como consecuencia que el lenguaje y la comunicación sean objetivos poco probables de la docencia universitaria en general. A su vez, es manifiesta la dificultad en el dominio de textos académicos por parte de los estudiantes indígenas (aunque no exclusivamente) y el uso de la lengua materna solo en situaciones coloquiales y en redes privadas sin presencia pública.

Los efectos de la inequidad cultural y lingüística en la educación superior se manifiestan en una política y proyecto universitario poco coherentes con la política oficial de la "interculturalidad para todos". Esto se traduce en escasas redes de información y de cooperación entre las universidades públicas y las universidades interculturales; así como poco financiamiento para la investigación longitudinal del multiculturalismo y del plurilingüismo en la universidad.

De igual manera, la falta de principios y estrategias interculturales metódicas en las reformas académicas y curriculares, la escasa identificación de identidades étnicas y de hablantes de lenguas indígenas en la universidad, y la insuficiente investigación científica y vinculación con las comunidades indomexicanas, que se realiza en algunos sectores de las ciencias sociales, revelan un desarrollo incipiente de la filosofía intercultural en la universidad (Muñoz, 2008).

En el ámbito académico es "normal", desde una lógica miserabilista (Jordan, 1998, p. 26) atribuir a factores culturales y lingüísticos la baja proficiencia cognitiva y comunicativa de los estudiantes indígenas, ubicarlos en un bilingüismo funcional no coordinado y desconocer los perfiles y desarrollo de habilidades lingüísticas, comunicativas y académicas de los mismos.

También, los sentimientos y razonamientos de exclusión y discriminación de los estudiantes indígenas se reflejan en los 
insuficientes diálogos y debates acerca de la diversidad etnolingüística y la comunicación intercultural dentro y fuera de las aulas; en las redes socioétnicas cerradas sin presencia pública; en el dominio de discursos epilingüísticos de lealtad etnolingüística (Boyer, 2009) y de vergüenza étnica; y en general en las creencias y deseos no siempre valorados respecto del cambio sociocultural (Muñoz, 2010a; Cruz, 2009).

De manera reciente, debido a que el enfoque intercultural en la educación básica no ha dado los frutos que se imaginaban, "los debates y trabajos evaluativos coinciden en cambiar la mirada y centrar la estrategia de la reforma educativa a la educación superior donde se están formando y preparando los recursos profesionales para el desarrollo global y competitivo de las naciones" (Muñoz, 2008, p. 5).

En ese contexto actualmente las acciones y estrategias impulsadas por el Estado mexicano son la creación de:

- instituciones indígenas de educación superior -como la Universidad Autónoma Indígena de México en Sinaloa, fundada en el periodo 1999-2001-(Rebolledo, 2005, p. 191), y recientemente las universidades interculturales (CGIB-SEP, 2006);

- programas o institutos dentro de universidades públicas o privadas (ANUIES-Fundación Ford, 2001);

- propuestas para crear programas o instituciones en regiones diversas como el proyecto de revitalización idiomática que estaría a cargo del Centro de Investigaciones y Estudios Superiores en Antropología Social, CIESAS (López, Moya \& Hamel, 2009, p. 256); y

- becas para estudios superiores con apoyo local e internacional (Ford Foundation International Fellowships Program, 2001), en la modalidad de estrategias de "acción afirmativa" (Fullinwider, 2009).

Aunque existen avances en la atención a estudiantes indígenas, las políticas educativas interculturales se focalizan en la normatividad jurídica y en las regulaciones de la vida académica universitaria. Por lo cual, "los principios de las políticas interculturales que adoptan algunas universidades reflejan en gran medida el proyecto 
institucional del desarrollo educativo, los cuales constituyen parámetros de contrastación con los razonamientos, valoraciones y prácticas de los estudiantes indígenas" (Muñoz, 2010b, p. 16). Ese es el tema principal de este artículo.

\section{Reflexividad sociolingüística: algunas características}

La reflexividad sociolingüística tiene antecedentes en la llamada teoría de las actitudes y las actitudes lingüísticas, esta última consolidada como una importante tradición académica en la sociolingüística y en la lingüística aplicada de las últimas seis décadas. Pero es en la psicología social, "específicamente en teorías sobre los prejuicios (Allport, 1935, 1954; Brown, 1998) y la cognición social (Bruner \& Tagiuri, 1954)" (Muñoz, 2010a, p. 75) desde donde la reflexividad social acerca del lenguaje cimienta sus bases explicativas. En este tipo de indagaciones la tarea principal consiste en "comprender las interpretaciones o representaciones de las personas (Dortier, 2004) -y de sus predisposiciones- acerca de diversas conductas asociadas con el lenguaje y la comunicación y de su subsecuente trato a los usuarios de tales comportamientos y formas" (Muñoz, 2010a, p. 75).

En México, estudios pioneros son los de Hans Saettele (1978), quien afirma que la reflexividad del lenguaje natural se debe principalmente a la unificación del contenido de una comunicación. En sus investigaciones identifica una reflexividad primaria y una secundaria. La primera abarca la comprensión de los sujetos entre sí y a nivel de experiencias mutuas; la segunda es un conjunto de conocimientos acerca del lenguaje y su conocimiento preconsciente, de carácter discontinuo y finito. Por una parte, la reflexividad secundaria es una condición de la toma de conciencia de los sujetos y de las restricciones impuestas a la reflexividad primaria; por otra, puede asumir un desarrollo parasitario.

Por su parte, Hamel y Muñoz (1988) caracterizan a la reflexividad como un mecanismo básico de comunicación y modelo de representaciones, factor y reflejo del proceso social en torno a la lengua o las lenguas de una comunidad de habla. Así mismo, recalcan 
que la reflexividad o el discurso reflexivo de los hablantes es una práctica interpretativa o racionalidad preteórica acerca de sí mismos o de las lenguas donde la condición indígena opera como un principio estructurante de la reflexividad del sujeto hablante (Muñoz, 1990).

En un estudio respecto de las distintas visiones acerca del fenómeno de la discriminación, muy latente en el país, Muñoz (2006) encuentra que la reflexividad en torno a la ontogénesis del desarrollo lingüístico, cultural e identitario de hablantes de lenguas indígenas en relación con la educación muestra las experiencias de violencia institucional y asimilación lingüística en la escolaridad primaria; la relación interétnica y el uso de estrategias y códigos panétnicos en el nivel de la escolaridad secundaria; las experiencias de abuso y discriminación por no emplear el español "correcto" en contextos urbanos (bachillerato) y discursos que reivindican la lengua y la cultura a partir de conocimientos técnicos y científicos (educación superior).

Recientemente Cruz (2009) desarrolló un estudio acerca de la orientación ética del discurso reflexivo de profesores bilingües indígenas. En dicha investigación, la reflexividad sociolingüística se caracteriza por referir sobre "sí mismo", "nosotros" y "los otros" en "situaciones o sucesos de satisfacción o euforia", "relativa relevancia o preeminencia" y "conflicto o brete". El autor sostiene que el discurso autobiográfico de los profesores se orienta hacia parámetros de historicidad, evaluación del origen y formulación de expectativas con respecto al futuro etnolingüístico, cuya característica principal son las explicaciones de interés, las argumentaciones cognitivas y valorativas y las declaraciones de intenciones o regulaciones de cambio sociocultural.

En suma, se puede decir que la reflexividad sociolingüística es un mecanismo básico de comunicación y modelo de representaciones, que funciona como factor y reflejo del proceso social en torno a la lengua o las lenguas de una comunidad de habla. Se trata, pues, de un mecanismo constitutivo, intencional y regulativo de la comunicación lingüística, que se expresa a través de representaciones cognitivas, razonamientos, normatividades, evaluaciones y descripciones de 
los recursos lingüísticos y socioculturales de los hablantes (Muñoz, 2010a).

\section{Metodología}

Primero se realizó una contextualización de las universidades que formaron parte de este estudio donde se revisaron las regulaciones multiculturalistas de la educación superior, se obtuvo información estadística de matrícula e inscripción a carreras y se exploraron de manera ejemplar algunas bibliotecas y otros acervos respecto de multiculturalismo y educación. Después se pidió la colaboración de estudiantes indígenas para la construcción de discursos autobiográficos (Cfr. Muñoz, 2010a; Cruz, 2010) y se aplicaron entrevistas a estudiantes, académicos y administradores.

En este trabajo se adopta un enfoque de análisis cualitativo de contenidos reflexivos en dos orientaciones (Cruz, 2009; Muñoz, 1983, 2010a; Ruiz, 1996). Primero, los temas y tópicos detectados se presentan de acuerdo con las dimensiones del desarrollo educativo intercultural formuladas por Muñoz $(1998,2002)$. Después, se propone un esquema de funcionamiento de las dimensiones señaladas que adquieren la forma de creencias, formatos de uso público, valoraciones y declaraciones de intenciones o regulaciones de cambio sociocultural (Cfr. Muñoz, 1983, 2010a, 2010b), expresados en diferentes niveles de intencionalidad (Acero, 2001; Dennett, 1996; Searle, 1991).

\subsection{Actores participantes}

Los resultados de esta investigación se derivan de discursos autobiográficos de 17 estudiantes de distintos semestres de la Licenciatura en Educación Primaria y Preescolar para el Medio Indígena de la UPN 201 (LEPEPMI'90) ${ }^{3}$ y 27 estudiantes de la

3 La Universidad Pedagógica Nacional se ha encargado desde 1982 de formar cuadros de profesores indígenas a través de sus programas de licenciatura escolarizada y semiescolarizada, pero es en la década de 1990 cuando la formación superior de maestros indígenas en servicio comienza en la modalidad de programas específicos no autónomos con la apertura de la Licenciatura en Educación Preescolar y Educación Primaria para el Medio Indígena (Nivón, 2009; Salinas y Avilés, 2003). 
UABJO de distintas carreras, varios de ellos afiliados a la Unidad de Atención Académica a Estudiantes Indígenas (UAAEI) y a la Casa del Universitario Indígena (CUI). Además se aplicaron 12 entrevistas a estudiantes y académicos de la LEPEPMI'90 y 21 entrevistas a personal directivo, académico, administrativo y estudiantes de la UABJO.

\subsection{Contexto de las universidades}

El estado de Oaxaca se ubica al sur del país en el extremo suroeste del istmo de Tehuantepec. Oaxaca está dividido en ocho regiones geográficas en las que se distribuye una rica composición multicultural y ecológica. De una cantidad total de 3.801 .962 personas que viven en el estado (INEGI, 2011a), 1.165.186 personas mayores de cinco años hablan alguna lengua indígena (INALI, 2008; INEGI, 201 lb), lo que representa el 34\% de la población (INEGI, 201 la). Por su parte la Ley de Derechos de los Pueblos y Comunidades Indígenas del Estado de Oaxaca (2001) reconoce la existencia de 15 pueblos indígenas en el territorio oaxaqueño: Amuzgos, Cuicatecos, Chatinos, Chinantecos, Chocholtecos, Chontales, Huaves, Ixcatecos, Mazatecos, Mixes, Mixtecos, Náhuatls, Triquis, Zapotecos y Zoques, distribuidos en 564 municipios, de los 570 existentes, localizados mayoritariamente en zonas rurales. Las lenguas más habladas en el estado de Oaxaca son las lenguas zapotecas, mixtecas, mazatecas y mixes.

\subsection{Los universos de estudio: la LEPEPMI'90 de la UPN y la UABJO}

La Universidad Pedagógica Nacional (UPN 201) es una institución de educación superior enfocada en la formación de profesionales de la educación y creada en 1978. El estado de Oaxaca cuenta con tres unidades: Oaxaca (201), Tuxtepec (202) y Ciudad Ixtepec (203). De los programas de formación ofertados se eligió para esta investigación a la Licenciatura en Educación Preescolar y Primaria Plan 1990 (LEPEPMI'90), la cual plantea en su perfil de ingreso que el estudiante presta sus servicios en el subsistema de educación indígena; su experiencia en la docencia es variada por el número de años de servicio, el contexto sociocultural y lingüístico en el que realiza su trabajo y el nivel educativo y grados escolares que atiende y funciones desempeñadas son variadas. 
Por otro lado, la Universidad Autónoma Benito Juárez de Oaxaca (UABJO) es una institución de educación superior y media superior creada el 8 de enero de 1827. Es conocida comúnmente como la "máxima casa de estudios de Oaxaca" y cuenta con 32 programas educativos que se agrupan por áreas de conocimiento. El perfil de ingreso de los programas de formación exige del aspirante conocimientos, destrezas, habilidades, actitudes y valores según el área de estudio.

\subsection{Algunos datos estadísticos}

La identificación de estudiantes de origen etnolingüístico, al igual que las experiencias de formación intercultural, son iniciales en la mayoría de las universidades públicas de modelo tradicional. Según algunas fuentes periodísticas, los formatos públicos más referidos señalan que solo el 1\% de jóvenes indígenas accede a la educación superior (Henríquez, 2012) años alrededor del 64\% solo tiene educación básica y únicamente el 2 o 3\% cuenta con instrucción superior (Pérez, 2012); y solo dos de cada 100 personas pertenecientes a un pueblo originario alcanzan el nivel universitario (Avilés, 2011).

En la exploración realizada de la LEPEPMI'90, según datos estadísticos de la promoción 2010, de un total de 791 aspirantes para cursar la licenciatura en alguna de las subsedes de la unidad Oaxaca de la UPN 201, 643 personas hablaban lengua indígena, de ellos 369 eran mujeres y 274 hombres. Escribían textos en lengua materna 338; 717 exponían que algún familiar hablaba lengua indígena y 518 manifestaban haber estudiado en educación bilingüe. Sin embargo, de un total de 285 aspirantes que presentaron examen para la unidad sede, solo fueron aceptados 87 profesores-estudiantes que representan el 30,5\%. Algo similar sucedió con los aspirantes aceptados en las subsedes en las cuales los porcentajes se sitúan lejos de satisfacer las demandas de ingreso a la educación superior (Tabla 1).

4 Otros estudios posteriores indican que seis de cada 100 estudiantes lograban iniciar sus estudios universitarios (Bastiani, 2006: 25). 
Tabla 1. Matrícula-LEPEPMI'90 promoción 2010

\begin{tabular}{l|c|c|c}
\hline $\begin{array}{l}\text { Unidad sede y } \\
\text { subsedes }\end{array}$ & Total de aspirantes & Aceptados & No aceptados \\
\hline Oaxaca & 285 & $87(30,5 \%)$ & $198(69,4 \%)$ \\
\hline Pochutla & 69 & $32(46,3 \%)$ & $37(53,6 \%)$ \\
\hline Cacahuatepec & 0 & 0 & 0 \\
\hline Huajuapan & 81 & $31(38,2 \%)$ & $50(61,7 \%)$ \\
\hline Tlaxiaco & 148 & $63(42,5 \%)$ & $85(57,4 \%)$ \\
\hline Jamiltepec & 86 & $34(39,5 \%)$ & $52(60,4 \%)$ \\
\hline Huautla & 89 & $31(34,8 \%)$ & $58(65,1 \%)$ \\
\hline Total & 758 & $278(36,6 \%)$ & $480(63,3 \%)$ \\
\hline
\end{tabular}

Fuente: Datos estadísticos de ingreso a la LEPEPMI'90 promoción 2010 (2011). Subdirección académica de la UPN 201.

A diferencia de la UPN 201, la información de la UABJO contempla datos estadísticos de los estudiantes indígenas durante siete ciclos escolares (2004 a 2010) (UABJO, 2010). En el ciclo escolar 2004-2005, de un total de 28.141 estudiantes, tres (0,01\%) son indígenas y en el ciclo escolar 2005-2006, de 19.197 estudiantes cuatro $(0,02 \%)$ son indígenas. Es en el ciclo 2006-2007 en el que la población indígena incrementa, pues de un total de 19.653, ahora 3.410 (17,35\%) son indígenas. Paradójicamente, es en 2006 cuando la UABJO se adhiere al Programa de Atención Académica a Estudiantes Indígenas en Instituciones de Educación Superior (PAEIIES), con la creación de la Unidad de Atención Académica a Estudiantes Indígenas (UAAEI). Esta tendencia continúa y se ve reflejada en los ciclos 20072008 y 2008-2009.

Sin embargo, aunque la matrícula escolar de estudiantes indígenas ha ido en aumento, la diferencia con el total de la población escolar es significativa pues de 104.715 estudiantes, solo 12.042 eran indígenas en los períodos 2004-2005 al 2008-2009.

Respecto de la lengua indígena, según datos del período escolar 2006 a 2010, de un total de 16.199 estudiantes indígenas, solo 1.005 $(6,2 \%)$ son reportados como hablantes de alguna lengua indígena. De ellos, 503 (3,1\%) son mujeres y 502 (3\%) son hombres. Aunque la tendencia de los datos nos permite predecir que la cantidad de 
estudiantes hablantes de lengua indígena se mantendrá estable, la información proporcionada no nos dice cuáles son las lenguas que se hablan ni cuáles son las que tienen mayor número de hablantes.

\section{Resultados}

A continuación se presentan los hallazgos obtenidos en este estudio.

\subsection{Desarrollo educativo intercultural en universidades oaxaqueñas}

Los discursos reflexivos de los diferentes actores exponen temas y tópicos acerca del desarrollo educativo intercultural en la universidad en cuatro niveles diferenciados: el primero de ellos se refiere a las políticas y proyectos educativos; el segundo recupera algunas expresiones sobre el sistema universitario; el tercero a las experiencias en relación con los modelos académicos y curriculares; y el cuarto a la reflexividad sobre las prácticas escolares (Muñoz, 1998). Se trata de una secuencia lógica de contenidos reflexivos en diferentes niveles de concreción que dan cuenta de la "cuestión indígena" en la universidad.

Un segundo asunto, es que los variados discursos respecto del desarrollo educativo intercultural permiten identificar un esquema de funcionamiento de la reflexividad de los actores que se manifiesta a partir de informaciones y formatos de uso público, valoraciones, declaraciones de intenciones y regulaciones de cambio sociocultural en diferentes órdenes de intencionalidad. La implicación principal de este esquema de funcionamiento es que sugiere las principales necesidades y demandas de los estudiantes indígenas, un corpus temático de las valoraciones más recurrentes en relación con la lengua y la cultura, así como una aproximación a las acciones que los estudiantes proponen o impulsan para remediar las desigualdades, las inequidades y las exclusiones. A continuación se revisa cómo se realizan estas funciones sobre el desarrollo educativo intercultural, enfatizando el nivel de las prácticas escolares. 


\subsection{Discusiones principales}

\subsubsection{Formatos de uso público: rutas estables de reflexividad}

La primera función del esquema mencionado alude a las informaciones y formatos de uso público instalados en la reflexividad de los estudiantes. Se trata de estructuras lógicas de razonamiento, que suelen tener una plataforma temática y ciertas predicaciones. Son estructuras discursivas de cognición, valoración y acción socialmente consensuadas y con cierta estabilidad, que se imponen de manera casi natural a las personas, muchas de las veces, sin ser mayormente cuestionadas. A continuación se exponen las que se identificaron en este estudio.

En primer lugar, en la dimensión del sistema ${ }^{5}$ los formatos que se identificaron en la UABJO se refieren a la necesidad del "apoyo familiar para continuar estudiando” (N23D4IVUABJO2012), el origen étnico como limitante para estudiar y la falta de preparación como característica de estas personas, contenidos que permean en las representaciones de la denominada sociedad mestiza (CDI, 2006). Otro ejemplo son las predicaciones acerca de la UAEEI, que de hecho es el dominio facultado para impulsar las acciones de base étnica en la universidad. Lo mismo sucede con formatos sobre la "diversidad cultural", que acotan su significado exclusivamente hacia el sector indígena.

En el nivel de los diseños programáticos, los formatos versan acerca de los contenidos o temas que se desarrollan en los cursos que ofrece la LEPEPMI'90. Uno de ellos es aquel que indica la existencia de una "educación bilingüe e intercultural" -tanto en la universidad como en el trabajo docente- que, aunque es un asunto que requiere mayor investigación empírica y el ensayo de nuevas modalidades de enseñanza y aprendizaje, no se deja de hablar respecto de algo cuya corporeidad aún se encuentra en progreso y que en ocasiones suscita polarizaciones en los sentidos y significados que se le asignan. Lo mismo sucede con las predicaciones acerca de la diversidad cultural

5 No se identificaron formatos sobre el nivel de las políticas y proyectos educativos en ambos universos de estudio. En la dimensión del sistema solo se identificaron formatos en los discursos reflexivos de los estudiantes de la UABJO. 
y lingüística que se traducen discursivamente en los diversos tipos de intercambio de experiencias en la universidad.

En relación con la formación en la práctica, los profesoresestudiantes indican que los planes y programas de estudio presentan objetivos distantes de las realidades escolares, y también mencionan dificultades en la "recepción" y adecuación de los mismos (descontextualización).

Por otra parte, en la UABJO las acciones de base étnica en la UAAEI asumen discursivamente la manifestación de la interculturalidad, tales como el intercambio académico, sociocultural y deportivo excluyendo las relaciones interculturales de facto. De hecho, en las entrevistas los diferentes actores inmediatamente aludían a la UAAEI como uno de los espacios diseñados para tal objetivo.

En la dimensión de las realidades escolares, los profesoresestudiantes de la LEPEPMI'90 destacaron temas como el bilingüismo, las funciones diversas del profesorado de educación indígena, el tipo de educación en el medio indígena, la situación socioeconómica y de marginalidad de las comunidades indígenas, la vida comunal y la reproducción de prácticas castellanizadoras en la escuela indígena. También se refirieron al futuro de las lenguas originarias -para algunos poco prometedor-, así como a las visiones que conciben a la identidad étnica como un ente puro e inmutable que es definido en las socializaciones tempranas.

En la UABJO, por el contrario, los formatos se relacionan con el carácter intercultural de los encuentros o espacios de intercambio académico, sociocultural y deportivo que impulsa la UAAEI respaldada por la CUI, así como la discriminación con motivos étnicos y de género (Tabla 2). 
Tabla 2. Formatos de uso público - LEPEPMI'90 y UABJO

\begin{tabular}{l|l}
\hline Temas & Estructuras de razonamiento estables \\
\hline $\begin{array}{l}\text { Distribución regular } \\
\text { e irregular de los } \\
\text { maestros }\end{array}$ & $\begin{array}{l}\text { Soy bilingüe, porque hablo las dos lenguas que son: el mixe como primera } \\
\text { lengua y el español como segunda lengua, pero en lo que se refiere a mi } \\
\text { práctica docente, no utilizo la lengua originaria ya que estoy desempeñando } \\
\text { mis labores en otra área lingüística, en la región de los coatlanes, en la sierra } \\
\text { sur de nuestro estado (...). (N3D4LEPEPMI90UPN2012)*. }\end{array}$ \\
\hline $\begin{array}{l}\text { El futuro de la } \\
\text { lengua }\end{array}$ & $\begin{array}{l}\text { En cuanto al futuro de las lenguas originarias creo que cada día hay menos } \\
\text { hablantes y si no concientizamos a las personas de que es importante } \\
\text { conservar nuestras lenguas irremediablemente desaparecerán, al ir } \\
\text { desapareciendo las lenguas iremos perdiendo nuestra identidad, nuestras } \\
\text { creencias, costumbres, etc., (N17D4LEPEPMI90UPN2012). }\end{array}$ \\
\hline $\begin{array}{l}\text { Discriminación en la } \\
\text { universidad }\end{array}$ & $\begin{array}{l}\text { l...) pues si ya con la condición de mujer nos encontramos en una "desventaja" } \\
\text { ahora mucho más siendo indígena, pues la mayoría de estas sufren las } \\
\text { consecuencias de vivir en un pueblo donde sus usos y costumbres las orillan } \\
\text { a hacer acciones como casarse a muy temprana edad, no poder estudiar } \\
\text { porque ellas tienen que ser las que atiendan los quehaceres del hogar, que } \\
\text { no participen en la política de sus pueblos, por mencionar solo unas (...). }\end{array}$ \\
(N25D4UABJO2012).
\end{tabular}

* Las notaciones al final de las citas corresponden al número de narrativa o entrevista: ( $N$ ); número de Dimensión (D); Programa de Formación (LEPEPMI'90) o Universidad (UABJO); y año del dato (2012).

\subsubsection{Valoración social de la lengua, la cultura y la educación}

La segunda función es aquella que se relaciona con la valoración del objeto del discurso reflexivo. Por valoración se entiende la tendencia favorable o desfavorable hacia un objeto significativo o sistema de creencias que puede implicar una toma de posición, la elección de ciertas alternativas entre muchas otras, o la orientación afectiva que puede imprimírsele a determinadas acciones y regulaciones. En la perspectiva que se adopta, la reflexividad sobre valoraciones de base hylética constituirá una de las principales fortalezas del enfoque de la reflexividad sociolingüística.

En la dimensión de política y proyectos educativos se presentan calificaciones que dan cuenta de las dificultades que los profesoresestudiantes de la LEPEPMI'90 de la UPN 201 viven en las realidades 
escolares al tomar como parámetro de comparación las políticas educativas oficiales. Así mismo, reconocen que falta desarrollar trabajo educativo en las comunidades y exponen que los derechos de los pueblos indígenas no son valorados ${ }^{6}$.

Por su parte, en la dimensión del sistema, que implica la organización y las estrategias del gobierno para ejecutar las doctrinas educativas oficiales, los estudiantes de la LEPEPMI'90 se refieren a las condiciones negativas en la profesión docente, la situación económica desfavorable, la experiencia en otros programas de formación y se reflexiona sobre el tránsito al nivel del sistema en la educación superior.

Por otro lado, los estudiantes de la UABJO reflexionan acerca de la continuidad de estudios al finalizar el nivel medio superior, la importancia del apoyo familiar para seguir estudiando y el apoyo de las becas que los estudiantes han recibido. En esta dimensión, las acciones de base étnica impulsadas por la UAAEI son valoradas positivamente, sin embargo, se expone que tales acciones no son impulsadas al nivel institucional ni están consolidadas.

En la dimensión de los modelos académicos de formación, los profesores-estudiantes de la LEPEPMI'90 destacan la influencia que han tenido los contenidos de los cursos en su preparación, los valores de convivencia y las relaciones establecidas principalmente con sus pares; y exponen las dificultades experimentadas con la lectura y la escritura académica. En cambio, como profesores, destacan la formación en la práctica, la recepción y adecuación de los planes y programas de estudio, así como los problemas que viven en la enseñanza y en el aprendizaje de sus alumnos.

En el caso de la UABJO, las prácticas de evaluación (memorización y repetición de temarios) y la experiencia académica vivida en las carreras elegidas es ampliamente referida. También se señalan positivamente los cursos y los Encuentros Interculturales impulsados por la UAAEI y se reconoce la importancia de la

6 No se identificaron valoraciones sobre la primera dimensión en los discursos autobiográficos de los estudiantes de la UABJO. 
lengua originaria, proponiéndose su rescate e inclusión en el nivel programático de las facultades, escuelas o institutos.

Por último, en la dimensión de las realidades escolares se recupera lo que los estudiantes de la LEPEPMI'90 realizan en sus espacios de adscripción laboral. La lengua materna se valora favorablemente, pero también es motivo de conflicto. Por último, se exponen aquellos factores que han incidido a favor de la identidad étnica, como lo es el emprender estudios universitarios o insertarse en el campo laboral.

En el contexto de la UABJO, se resalta la discriminación vivida en la universidad, principalmente por el género, procedencia indígena y uso de la lengua materna. En segundo lugar, se recuperan las experiencias de fortalecimiento académico y cultural en la CUI, así como el uso de la lengua indígena y las prácticas y valores de convivencia impulsados. Por último, se adelantan algunos de los posibles caminos que los estudiantes seguirán al egresar de la universidad. A continuación, se muestran algunos discursos con orientación valorativa de los estudiantes de las universidades de estudio (Tabla 3).

Tabla 3. Discursos valorativos de la LEPEPMI'90 y la UABJO

\begin{tabular}{l|l}
\hline Temas & Calificaciones, comparaciones, estimaciones, etc. \\
\hline $\begin{array}{l}\text { Transmisión } \\
\text { intergeneracional } \\
\text { de la lengua }\end{array}$ & $\begin{array}{l}\text { (...) no parecen tener actitud de fortalecimiento de za, parece no } \\
\text { importarles, ija, y a mí sí me debe! Bien, de acuerdo aunque no tengo el } \\
\text { tiempo. (N5D4LEPEPMI90UPN2012). }\end{array}$ \\
\hline $\begin{array}{l}\text { El futuro de la } \\
\text { lengua }\end{array}$ & $\begin{array}{l}\text { En cuanto al futuro de las lenguas originarias creo que cada día hay menos } \\
\text { hablantes y si no concientizamos a las personas de que es importante } \\
\text { conservar nuestras lenguas irremediablemente desaparecerán, al ir } \\
\text { desapareciendo las lenguas iremos perdiendo nuestra identidad, nuestras } \\
\text { creencias, costumbres, etc. (N17D4LEPEPMI90UPN2012). }\end{array}$ \\
\hline $\begin{array}{l}\text { Discriminación en } \\
\text { la universidad }\end{array}$ & $\begin{array}{l}\text { (...) durante los estudios hubo ocasiones en las que algunos de los } \\
\text { compañeros del grupo mostraban actitudes discriminatorias, como } \\
\text { miradas de menosprecio o presuntuosamente hacia un pequeño grupo de } \\
\text { jóvenes provenientes de comunidades indígenas entre ellos yo, ante esto al } \\
\text { inicio nos molestaba ya después hacíamos caso omiso y tratábamos de que } \\
\text { no nos afectara (...). (N24D4UABJ02012). }\end{array}$ \\
\hline $\begin{array}{l}\text { La Casa del } \\
\text { Universitario } \\
\text { Indígena (CUI) }\end{array}$ & $\begin{array}{l}\text { (...) conocía personas que como yo también llegaron por falta de economía } \\
\text { esta casa, de esta familia, de este grupo (...). (N27D4UABJ02012). }\end{array}$ \\
\hline
\end{tabular}




\subsubsection{Regulaciones de cambio sociocultural en la universidad} Un tercer elemento del esquema de funcionamiento del desarrollo educativo intercultural son las regulaciones de cambio sociocultural que promueven actores institucionales y estudiantes para enfrentar las desigualdades, las inequidades y las exclusiones. Son estrategias con orientación sociocultural que aparecen en los instrumentos jurídicos y reglamentarios y, por otro lado, son normas de uso que tienen la forma de patrones de interacción o discursos que se estandarizan en las relaciones sociales, las cuales pueden ser lingüísticas, retóricas, discursivas, etc. Una muestra de este tipo de discursos reflexivos refiere esfuerzos explícitos realizados por la universidad y por los mismos estudiantes para cambiar el presente y el futuro de las lenguas y cultura indígenas; por otra parte, son enunciados sobre una serie de actividades, que en el mejor de los casos, quedan en el despliegue discursivo de buenas intenciones.

Para el caso de la UPN 201, en la primera dimensión descrita, una de las actividades que se propone para desarrollar la lengua indígena es el trabajo en y con las comunidades. Este tipo de acción -trazada de manera genérica-en las dimensiones siguientes adquiere forma de tal manera que se pueda alcanzar dicho objetivo ${ }^{7}$.

En la segunda dimensión, la experiencia escolar de los estudiantes de la LEPEPMI'90 se relaciona con aquellos tópicos que señalan que "existe la clara necesidad de ayudar a los alumnos de las comunidades más alejadas de la ciudad para conocer su exterior" (N1D2LEPEPMI90UPN2012), por lo tanto se propone que primero habrá de enseñarse lo que hay en la comunidad para después salir fuera. También se plantea que para que los estudiantes encuentren condiciones favorables para su desarrollo en el tránsito por la universidad es prioritario que los espacios formativos se sensibilicen ante la diversidad. Otro ejemplo de acciones, plantea a los profesores-estudiantes la tarea de prepararse, "porque hay retos importantes dentro del aula, para desenvolverse de una forma adecuada" (N3D2LEPEPMI90UPN2012), en todo caso, se asume que

7 No se identificaron regulaciones en torno a la primera dimensión en los discursos autobiográficos de los estudiantes de la UABJO. 
lo aprendido en la universidad deberá llevarse a la práctica docente. Por último, como estudiantes, exponen la necesidad de que en la formación académica los asesores establezcan un vínculo entre las discusiones teóricas y la práctica para analizar la cotidianidad del trabajo áulico.

Por otro lado, en la UABJO se enuncian una serie de acciones de base étnica, como la impartición de cursos (extracurriculares, preventivos y remediales), intercambios académicos, encuentros de estudiantes, eventos interculturales, obligatoriedad en la enseñanza de la lengua indígena, servicios de cómputo, tutorías y asistencia a congresos. La mayoría de ellas asociadas a las actividades que impulsa la UAAEI.

Respecto de la dimensión de los diseños curriculares, se remarca la valoración de los contenidos o temas de los cursos abordados en la LEPEPMI'90. Se trata de planteamientos genéricos con intencionalidad valorativa sobre el respeto a las diferentes formas de vida en las comunidades indígenas y la preservación-inclusión de rasgos y saberes culturales al trabajo académico en la universidad.

Un hallazgo importante en esta discusión son las propuestas desde abajo y hacia dentro de la universidad. En esta perspectiva se sugiere la certificación de la lengua materna para efectos de titulación, convenios de colaboración con las comunidades indígenas para el diseño e implementación de programas enfocados en la preservación de las lenguas maternas y cursos donde los estudiantes que sepan hablar una lengua materna les enseñen a sus coetáneos. Aunque la mayoría de las acciones que se proponen siguen direcciones parecidas, la distinción se establece porque las acciones que vienen desde abajo proponen una interlocución simétrica y horizontal con sus pares no indígenas, como es el caso de la enseñanza de la lengua indígena a aquellos que se interesen en aprenderla, sin embargo, las posibilidades de actuación son desfavorables como se pudo constatar en los discursos de los estudiantes.

En la última dimensión los profesores-estudiantes de la UPN 201 exponen algunas de las acciones que realizan e impactan 
los espacios en los que se encuentran laborando. Es lo que se ha denominado como gestión en los dominios de inserción laboral, es decir, son acciones desde afuera que bajo ciertas condiciones tienen resonancia o son tema de reflexión hacia dentro de la universidad. Aunque no es un caso típico y generalizable, los diferentes discursos reflexivos respaldan los argumentos. Algunos ejemplos de este tipo de acciones son el caso de una profesora que colabora en el diseño y grabación de programas de radio comunitaria; el papel que juegan los profesores-estudiantes en distintas funciones académicas, directivas y sindicales; el rol que desempeñan como autoridades municipales; la promoción y difusión de eventos en las comunidades de adscripción, en las zonas escolares y fuera de ellas; al nivel programático y del trabajo en el aula, la recuperación del contexto de la comunidad y los materiales del medio. Así mismo, se sugiere la "asimilación e integración a la vida comunal” (N6D4LEPEPMI90UPN2012).

Respecto de la educación bilingüe se reportan experiencias donde los profesores echan mano de las dos lenguas que hablan, tanto para apoyar a los niños que no hablan la lengua oficial, así como para ayudar a aquellos cuya lengua es el español. Se trata, en todo caso, de establecer interlocuciones de entendimiento posible (Rodríguez y Rivera, 1996). En el extremo opuesto se ubican aquellas experiencias que denuncian la irregular distribución de los profesores en zonas donde la lengua del docente no corresponde al lugar de adscripción. Para cerrar este apartado, al nivel de las realidades escolares en la UABJO, la discriminación vivida en la universidad se sobrellevará a partir de prácticas y estrategias (Chávez, 2008) que, en el mejor de los casos, se vuelven una situación incómoda y recíproca con los estudiantes no indígenas. En cambio, desde la perspectiva de los estudiantes que habitan en la CUI, se manifiesta que el impulso de la cultura se ha dado a partir de la asistencia a los encuentros interculturales y que la lengua se fortalece por la convivencia entre personas de diferentes etnias que permiten el aprendizaje de las mismas, aunque sea de manera muy incipiente. En síntesis, se trata de propuestas que necesitan mayor involucramiento de los estudiantes para impulsar desde abajo la voz y prácticas de un sector históricamente excluido, pero que ha sabido sobrellevar ese lastre (Tabla 4). 
Tabla 4. Metodologías de cambio sociocultural - LEPEPMI'90 y UABJO

\begin{tabular}{l|l}
\hline Temas & Regulaciones o declaración de intenciones \\
\hline $\begin{array}{l}\text { Valoración social de la } \\
\text { lengua indígena en el } \\
\text { aula y fuera de ella. }\end{array}$ & $\begin{array}{l}\text { (...) lo que hice fue hablar con los niños más que con los papás y/o } \\
\text { mamás, en la intención de que valoren el idioma de sus abuelos, y que } \\
\text { quienes saben un poco que lo fortalezcan. (N5D4LEPEPMI90UPN201). }\end{array}$ \\
\hline $\begin{array}{l}\text { Lengua indígena } \\
\text { y el contexto } \\
\text { comunitario: algunas } \\
\text { interrelaciones. }\end{array}$ & $\begin{array}{l}\text { Por lo que me resta seguir preparándome más tanto en la escuela como } \\
\text { en la vida misma, en sí, asimilar e integrarme a la vida comunal, ya que } \\
\text { de ahí puedo darle realmente un significado a mi vida y, por ende, a mi } \\
\text { trabajo, reorientar mis objetivos. (N6D4LEPEPMI90UPN2012). }\end{array}$ \\
\hline $\begin{array}{l}\text { Fortalecimiento } \\
\text { académico y cultural en } \\
\text { la CUI. }\end{array}$ & $\begin{array}{l}\text { (...) las acciones que se han realizado para impulsar la cultura, todos } \\
\text { asistimos a encuentros interculturales (...). (N40D4UABJ02012). }\end{array}$ \\
\hline $\begin{array}{l}\text { Discriminación en la } \\
\text { universidad. }\end{array}$ & $\begin{array}{l}\text { (...) nos mostrábamos apáticos o simplemente no intentábamos } \\
\text { integrarnos, situación incómoda y recíproca con el resto del grupo. } \\
\text { (N24D4UABJ02012). }\end{array}$ \\
\hline
\end{tabular}

\subsubsection{Niveles de intencionalidad de los discursos reflexivos}

Para culminar este texto, a continuación se presenta lo que se ha denominado como niveles intencionales del discurso reflexivo (Cruz, 2009; Muñoz, 2010a). El principal reto de esta discusión es mostrar si existe la posibilidad de que los estudiantes cuestionen o juzguen creencias y deseos arraigados que circulan en la escena universitaria sobre el desarrollo de la lengua, la cultura y las acciones educativas orientadas a atenderlos. De ello depende, en gran medida, la posibilidad de gestar diseños intencionales creativos para hacer frente a las inequidades, las desigualdades y las exclusiones.

Una discusión central derivada de los argumentos anteriores es que los diseños intencionales basados en normas y acciones institucionales sugieren con cierta fascinación, la creencia de que las metodologías de cambio sociocultural son suficientes y, por lo mismo, ideales para afrontar los conflictos y demandas de los estudiantes. No es casualidad que hoy día se establezcan acciones destinadas a atender las necesidades de estos estudiantes en los diferentes espacios de la universidad, sin embargo, pesa el hecho de que el grueso de la comunidad universitaria y la sociedad en general no se involucren en una empresa que incumbe a todos.

Ejemplos de discurso intencional de orden 1 son aquellos en el que se hacen declaraciones basadas en formatos de uso público 
que dan la impresión de sustentarse en un conocimiento claro del fenómeno a explicar. Sin embargo, como se ve en el testimonio siguiente, el estudiante hace una declaración sobre el bilingüismo y su asociación con la Ley de los Derechos Lingüísticos que revela un conocimiento indefinido:

Como estudiante y, bueno por la cuestión de, del bilingüismo, por, ehh, me entero pues de que ya existe la Ley de los Derechos Lingüísticos, acuerdos con la OIT, entonces de que políticamente hemos avanzado. (E29LEPEPMI902011).

En cambio, un discurso reflexivo con intencionalidad de nivel 2 es aquel en el que se cuestionan creencias basadas en informaciones del nivel hylético o de primer orden. Este nivel también se refiere a aquellos razonamientos que utilizan selecciones normativas o legales para fundamentar los propios razonamientos, como se observa en el siguiente testimonio:

Que uno de los derechos de los pueblos y las comunidades indígenas que reconoce el apartado "A" del artículo 2o. de nuestra Ley Suprema es el de "la libre determinación y, en consecuencia, el de la autonomía para preservar y enriquecer sus lenguas, conocimientos y todos los elementos que constituyen su cultura e identidad" (Instituto Nacional de las Lenguas Indígenas. 2008, p.1), cosa que no aparece en el plan y programa de estudio. (N4D1LEPEPMI90UPN2012).

En la dimensión del sistema, algunos discursos de intencionalidad de orden 1 exponen como válidos determinados razonamientos que justifican la "decisión" de estudiar en la UPN. Declaraciones acerca de la identidad étnica, hablar una lengua indígena y poseer un conjunto de conocimientos endógenos son empleados enfáticamente, dejándose de lado otras posibilidades de explicación:

Pues, primeramente, ser, ser chatino, yo soy chatino de la región de Juquila, soy de Santiago Yeitepec, hablante de esta misma lengua, ingreso a esta unidad por lo mismo de que

8 Las notaciones al final de las citas corresponden al número de narrativa o entrevista (N/E); Académico (A), Estudiante (E), Bibliotecario (B) y las siglas de otros actores; Programa de Formación (LEPEPMI'90) o Universidad (UABJO); y año del dato (2012). 
me dan las facilidades de estudiar realmente cómo son mis indígenas, cómo son los chatinos allá, y de traer lo poco que obtengo allá a esta universidad y comparar tanto lo educativo es este nivel académico que es la universidad como también estamos relacionando, trabajando la práctica docente en el campo ¿no? (E26LEPEPMI902011).

En contraparte, un discurso de intencionalidad 2 con respecto al perfil de los profesores, la preparación que poseen y su experiencia escolar abre paso al cuestionamiento del porqué no usan la lengua indígena en la escuela así como se reconoce la vulnerable cultura académica de sí mismo.

(...) todo el trabajo que efectuaba lo realizaba con ideas de lo que debía de hacer pero no lo hacía, situación que me ponía en conflicto conmigo mismo pues en el fondo me sentía mal por inventar cosas que no realizaba. (N7D2LEPEPMI90UPN2012).

En el caso de la UABJO, los tópicos en los que se presentan diseños intencionales de orden 1 y 2 son los concernidos a la formación de los estudiantes etnolingüísticamente diferenciados, el apoyo familiar para seguir estudiando y aquellas valoraciones sobre la educación en contextos urbanos. En los siguientes casos, un recurso trascendental que cuestiona los diseños de base es el uso del discurso citado y la voz reflexiva de los estudiantes que otorga validez a los razonamientos propios:

(...) junto con mis amigos nos poníamos a pensar qué sería de nosotros cuando fuéramos grandes, algunos ambicionaban ser grandes empresarios, otros ser profesionales en alguna carrera y unos más solían decir "yo no sé para qué estudio si me voy a quedar a trabajar en el campo". (N27D2UABJO2012).

En el siguiente nivel, asuntos relacionados con el "ser docente del medio indígena", las dificultades al planear y el poco éxito en la aplicación de estrategias de enseñanza, constituyen ejemplos de intencionalidad de orden 2 que cuestionan los métodos de instrucción utilizados. Se invocan asuntos sobre la formación en la práctica y se reprueban acciones como lograr la disciplina en el aula a través de castigos: 
Así fue pasando mi educación primaria y ahora veo que los métodos eran los equivocados por lo que creo que en mis manos está para que ya no vuelva suceder lo mismo con mis alumnos y no vaya llegar un momento en que algún alumno vaya a dejar la escuela solo por esos métodos. (N11D3LEPEPMI90UPN2012).

En la UABJO, uno de los ejemplos en el que se cuestionan creencias de orden 1 de intencionalidad tiene que ver con la valoración de la lengua indígena empleando el recurso de discurso citado. Se trata de un problema que alude al estatus de las lenguas indígenas en el país, las cuales a pesar del reconocimiento legal no logran instalarse en los proyectos de las universidades públicas en los diferentes niveles del desarrollo educativo intercultural:

(...) un claro ejemplo es que para poder titularse en casi todas las carreras se necesita una certificación del inglés, que si es importante esta lengua no lo puedo negar pero más aún es nuestra lengua, porque ellas son nuestra raíz, dice un filósofo: "nadie puede dirigirse en la vida sin saber cuál fue su historia" y es muy cierto para poder crearse futuro, debemos conocer quiénes fuimos primero. (N25D3UABJO2012).

En la dimensión de las prácticas, la función de profesor de educación indígena, las dificultades para trabajar la educación bilingüe y el aprendizaje de la lengua indígena, sugieren ejemplos en los que se reconocen los diferentes roles que desempeña un profesor y la subordinación de la lengua indígena al español a consecuencia de la discriminación:

(...) luego entonces me di cuenta de que la labor del docente no se centraba solo en enseñar cosas sino que a veces teníamos que hacerle de doctor, de psicólogo y hasta a veces de padres, porque como un grupo escolar es muy diverso y cada uno de los niños tiene diferentes necesidades. (N10D4LEPEPMI90UPN2012).

Por otra parte, en la UABJO se detectaron algunos discursos reflexivos que aluden principalmente al fenómeno de actitudes discriminatorias hacia los estudiantes indígenas y la mujer indígena, así como a la vergüenza de hablar la lengua originaria. Profundizando 
en el tema de la lengua indígena, a continuación se presentan discursos de nivel 1 y 2 de intencionalidad para ejemplificar este asunto:

Entonces dado la discriminación que había de escuchar alguna otra lengua, mi madre no nos enseñó porque mi papá no le dio permiso que hablara y que nos enseñara. Sin embargo, tengo la oportunidad de escuchar hablar a mis tíos, abuelos con mis primos. Tengo cierto conocimiento de entender la lengua y bueno, me agrada mucho, sin embargo, tengo toda la intención de aprenderla más, pero el tiempo y también la disposición no ha hecho posible que logre comprenderlo en su totalidad. El(4ICEUABJO2011).

Me parece verdaderamente triste cómo nuestras lenguas maternas se están extinguiendo solo por la vergüenza de la mayoría de los jóvenes a hablarla, en la escuela he tenido muchos conocidos que les da pena decir que hablan una lengua indígena solo por el miedo al rechazo, yo digo que deberían sentirse orgullosos porque hablan una lengua, a mi parecer también se da esto porque se da más importancia a otras lenguas que a las maternas (...) (N25D2UABJO2012).

En resumen, los discursos reflexivos reportan un panorama que comienza a ceder paso a la valoración y cuestionamiento de los discursos y prácticas que provienen desde la cima de la administración y academia universitarias o que circulan en los contextos en los que se desenvuelven los estudiantes. Es decir, se comienzan a analizar creencias y acciones que supondrían una redefinición de las relaciones interculturales en los dominios universitarios y sociales, principalmente en los espacios de adscripción de los profesoresestudiantes de la LEPEPMI'90 y en las acciones desde abajo y hacia dentro por parte de los estudiantes de la UABJO, especialmente de la CUI.

\section{Conclusiones}

El futuro de los estudiantes de origen etnolingüístico es una realidad en ebullición con muchos intereses en torno suyo (Muñoz, 2010a). En primer lugar las regulaciones de cambio asociadas con la diversidad y la comunicación intercultural se encuentran en una fase de instalación que no contempla al grueso de la sociedad por estar orientadas a 
una población en específico. En segundo lugar, las prácticas de gestión en favor de la lengua y la cultura por parte de los actores del sistema educativo siguen reducidas a espacios diseñados ex profeso, lo cual limita enormemente la participación activa de la comunidad estudiantil en general. En este caso fue evidente que las prácticas lingüísticas y cotidianas reportadas se circunscriben a espacios privados sin presencia pública o remitidos a algunos dominios y eventos universitarios. En tercer lugar, la reflexividad sociolingüística sobre la trayectoria académica de estos estudiantes muestra un camino lleno de dificultades, pero también de logros obtenidos principalmente en los espacios de inserción laboral para el caso de los profesoresestudiantes de la UPN 201 y en las experiencias desde abajo y hacia dentro impulsadas por los estudiantes universitarios de la UABJO principalmente de la CUI.

Los principales cambios o transiciones que se experimentan en las universidades que participaron de este estudio seguirán el camino de las acciones de base étnica, comúnmente denominadas "acciones afirmativas" (UABJO). Por el otro lado, existe una gran necesidad de indagar el nivel de las realidades en el campo laboral de los profesores-estudiantes para conocer más de cerca las distintas prácticas de autogestión a favor de la lengua y cultura indígenas que emanan en los discursos reflexivos (UPN 201). Esto se debe a que el espacio de acción docente acapara el tiempo y el esfuerzo de estos estudiantes en un momento coyuntural de discursos y acciones a favor de la diversidad lingüística y cultural en la escena planetaria.

Se concluye que hace falta una mayor presencia e impulso a las actividades más allá de los espacios áulicos y de la comunicación en situaciones rituales o privadas en las universidades. No es suficiente pregonar la presencia de una autoestima favorable o el orgullo de ser hablante de una lengua originaria. Es necesario crear circuitos de intercambio comunicativo, de difusión y promoción del desarrollo de la lengua y la cultura en los distintos dominios de la universidad que no solo se remitan a ceremonias y festividades.

Las producciones reflexivas en este sentido contribuyen a exponer una serie de temas y tópicos que debieran problematizarse 
en el recinto universitario, para pensar en condiciones de posibilidad y desarrollo. Es necesaria que la experiencia de estos estudiantes sea recuperada en políticas efectivas que tracen rutas académicas, pedagógicas y de investigación que profundicen en la comprensión que afecta a las lenguas y culturas indígenas. Además es una prioridad que estos contenidos lleguen a todos los actores del sistema universitario para pensar en acciones que no partan de las suposiciones de los diseñadores de políticas públicas. Indudablemente que el nivel de los modelos curriculares y académicos, así como la dimensión de las realidades áulicas constituyen los espacios más endebles y de escasa documentación.

Un asunto pendiente en la reflexividad de los estudiantes se relaciona con formas alternativas de construir el futuro deseable para los universitarios y comunidades indígenas. Eso es, nociones como autonomía, autodeterminación, territorio, nación y ciudadanía. Tal vez, esos sean temas de discusión y reflexión interlingüe e intercultural entre personas de origen diferente que faltan para orientar las políticas educativas interculturales.

Esta empresa requiere la exploración de cuatro componentes constitutivos de la realidad educativa y social de México. El análisis de las políticas multiculturales y plurilingüísticas de la universidad, la caracterización de la proficiencia lingüística y desempeño académico y convergente de los estudiantes; el estudio de la reflexividad y prácticas comunicativas de estudiantes indígenas y de otros sectores universitarios y el reconocimiento de una suerte de trayectoria etnolingüística (Muñoz, 2008).

En fin, se perciben esfuerzos para remediar las desigualdades e inequidades en los universos de estudio, sin embargo, los principios y filosofía de una "interculturalidad para todos" (Schemelkes, 2003) se encuentran distantes de los cambios que se pregonan, pues cada quien hace lo que puede sin lograr hasta el momento una vinculación entre los diferentes actores del sistema educativo. Es muy probable que en un momento en que las identidades étnicas y las lenguas son parte del universo gnoseológico de las personas, existan mejores posibilidades para iniciar un movimiento de redefinición de la universidad desde 
las bases de la población universitaria, aunque las exclusiones y las formas de discriminación siguen latentes.

\section{Referencias}

Acero, J. J. (2001). El lenguaje y el origen de la intencionalidad. En M. del C. Paredes (Ed.), Mente, conciencia y conocimiento (pp. 29-54). Salamanca: Ediciones Universidad de Salamanca.

Asociación Nacional de Universidades e Instituciones de Educación Superior, ANUIES y Fundación Ford. (2001). Programa de apoyo a estudiantes indígenas en instituciones de educación superior. Recuperado de http:// paeiies.anuies.mx/public/index.php?pagina=info_gral.html.

Avilés, K. (2011). De cada 100 indígenas dos llegan a la educación superior: expertos. La Jornada, p. 42. Recuperado de http://www.jornada.unam. $\mathrm{mx} / 2011 / 08 / 10 /$ sociedad/042n1soc

Badillo, J. (2008). La operación de los programas de tutorías en la Universidad Veracruzana y sus efectos en la experiencia escolar (Tesis de maestría inédita). Universidad Veracruzana, Facultad de Pedagogía, Xalapa, México.

Badillo, J., Casillas, M., y Ortiz, V. (2008). Políticas de atención a los grupos indígenas en la educación superior mexicana: el caso de la Universidad Veracruzana. Cuadernos Interculturales, 10, 33-61.

Bastiani, J. (2006). La educación intercultural universitaria: situación actual y desafíos. En T. Vilà (Coord.), Lengua, interculturalidad e identidad (pp. 23-28). Girona: Documenta Universitaria.

Boyer, H. (2009). Contactos y conflictos de lenguas: aproximación sociolingüística a las configuraciones de tipo diglósico con atención particular a los casos de Cataluña, de Galicia y del Paraguay. Signos Lingüísticos, 10, 9-32.

Chávez, M. E. (2008). Ser indígena en la educación superior. ¿Desventajas reales o asignadas? Revista de la Educación Superior, 37(148), 31-55.

Comisión Nacional para el Desarrollo de los Pueblos, CDI. (2006). Percepción de la imagen del indígena en México: diagnóstico cualitativo y cuantitativo. México D.F.: Autor.

Coordinación General de Educación Intercultural y Bilingüe, CGEIB Secretaría de Educación Pública, SEP. (2006). Universidad Intercultural. Modelo educativo. México D.F.: Autor. 
Cruz, G. (2009). El diseño ético de la intencionalidad en narrativas autobiográficas: un acercamiento desde la reflexividad sociolingüística (Tesis de maestría inédita). Universidad Pedagógica Nacional, México.

Cruz, G. (2010). El diseño ético de la intencionalidad en narrativas autobiográficas: un acercamiento desde la reflexividad sociolingüística. En M. Coronado y P. Mena (Coord.), Lengua y cultura en procesos educativos: investigaciones en Oaxaca (pp. 133-150). México D.F.: Universidad Pedagógica Nacional, Unidad Oaxaca.

Dennett, D. (1996). Kinds of minds. Toward an understanding of consciousness. New York: Basic Books.

Didou, S. y Remedi, E. (2006). Pathways to higher education: una oportunidad de educación superior para jóvenes indígenas en México. México D.F.: Asociación Nacional de Universidades e Instituciones de Educación Superior, ANUIES y Centro de Investigación y de Estudios Avanzados del Instituto Politécnico Nacional, CINVESTAV.

Didou, S. y Remedi, E. (2009). Los olvidados: acción afirmativa de base étnica e instituciones de educación superior en América Latina. México D.F.: Juan Pablos Editor y Centro de Investigación y de Estudios Avanzados del Instituto Politécnico Nacional, CINVESTAV.

Dietz, G. (2012). Reflexividad y diálogo en etnografía colaborativa: el acompañamiento etnográfico de una institución educativa "intercultural" mexicana. Revista de Antropología Social, 21, 63-91. http://dx.doi.org/10.5209/rev_raso.2012.v21.40050

Ford Foundation (2001). International Fellowships Program. Recuperado de http://fordifp.net/AboutIFP.aspx

Fullinwider, R. (2009). Affirmative action. En E. N. Zalta (Ed.). The Stanford encyclopedia of philosophy. Recuperado de http://plato.stanford.edu/ archives/win2009/entries/affirmative-action/

Gobierno del Estado De Oaxaca (1998). Ley de Derechos de los Pueblos y Comunidades Indígenas del Estado de Oaxaca. Recuperado de http:// www.oaxaca.gob.mx/lxi/info/legislacion/029.pdf

Hamel, R. y Muñoz, H. (1988). Desplazamiento y resistencia de la lengua otomí: el conflicto lingüístico en las prácticas discursivas y la reflexividad. En R. Hamel, Y. Lastra, y H. Muñoz (Eds.), Sociolingüística latinoamericana (pp. 101-146). México D.F.: Universidad Nacional Autónoma de México, UNAM.

Henríquez, E. (2012). Ser indígena en México aún representa exclusión y analfabetismo, señala Narro. La Jornada p. 16. Recuperado de http:// www.jornada.unam.mx/2012/10/27/politica/016n2pol 
Instituto Nacional de Estadística y Geografía, INEGI. (2011a). Panorama sociodemográfico de México. Censo de Población y Vivienda 2010. México D.F.: Autor.

Instituto Nacional de Estadística y Geografía, INEGI. (2011b). Principales resultados. Censo de Población y Vivienda 2010. México D.F.: Autor.

Instituto Nacional de Lenguas Indígenas, INALI. (2008). Catálogo de lenguas indígenas nacionales. Variantes lingüisticas de México con sus autodenominaciones y referencias geoestadísticas. México D.F.: Autor.

Jordan, J. A. (1998). La escuela multicultural. Un reto para el profesorado. Barcelona: Paidós.

López, L., Moya, R., y Hamel, R (2009). Pueblos indígenas y educación superior en América Latina y El Caribe. En L. López (Ed.), Interculturalidad, educación y ciudadanía. Perspectivas latinoamericanas (pp. 221-290). La Paz: Fundación para la Educación en Contextos de Multilingüismo y Pluriculturalidad, FUNPROEIB Andes y Plural Editores.

Mato, D. (Ed.). (2008). Diversidad cultural e interculturalidad en educación superior: experiencias en América Latina. Caracas: Instituto Internacional de la Unesco para la Educación Superior en América Latina y el Caribe, IESALC.

Mato, D. (Ed.). (2009). Instituciones interculturales de educación superior en América Latina. Procesos de construcción, logros, innovaciones y desafíos. Caracas: Instituto Internacional de la Unesco para la Educación Superior en América Latina y el Caribe, IESALC.

Muñoz, H. (1983). ¿Asimilación o igualdad lingüística en el Valle del Mezquital? Nueva Antropología, 22, 25-64.

Muñoz, H. (1990). El discurso indígena del conflicto lingüístico: una aproximación desde la reflexividad sociolingüística. Acciones Textuales, $1,143-162$.

Muñoz, H. (1998). Los objetivos políticos y socioeconómicos de la educación intercultural bilingüe y los cambios que se necesitan en el currículo, en la enseñanza y en las escuelas indígenas. Revista Iberoamericana de Educación, 17, 31-50.

Muñoz, H. (2002). La diversidad en las reformas educativas interculturales. Revista electrónica de investigación educativa, 2(4), 1-22. Recuperado de http://redie.uabc.mx/index.php/redie/article/view/58/105.

Muñoz, H. (2006). Niveles y tópicos de reflexividad sociolingüística a través de autobiografías. Signos Lingüísticos, 3, 69-94. 
Muñoz, H. (2008). Dominio lingüístico y académico de estudiantes bilingües indígenas en algunas universidades de México: habilidades comunicativas y académicas, reflexividad sociolingüística y políticas interculturales del lenguaje. Proyecto Conacyt 99274. México D.F.: UAM- Iztapalapa y Conacyt.

Muñoz, H. (2010a). Reflexividad sociolingüística de hablantes de lenguas indígenas: concepciones y cambio sociocultural. México D.F.: Ediciones del Lirio y Universidad Autónoma Metropolitana, UAM-Iztapalapa.

Muñoz, H. (septiembre, 2010b). Estudiantes indígenas y la gestión multicultural y plurilingüística de la educación superior. Trabajo presentado en el IV Seminario Internacional: Fronteiras étnicos-culturais e fronteiras da exclusão. Campo Grande, Brasil.

Nivón, A. (2006). La UAAEI en la Universidad Pedagógica Nacional. En Asociación Nacional de Universidades e Instituciones de Educación Superior, ANUIES (Ed.), Experiencias de atención a estudiantes indígenas en instituciones de educación superior (pp. 19-38). México D.F.: Autor.

Nivón, A. (2009). Formación de educadores indígenas en la Universidad Pedagógica Nacional. Recuperado de http://www.educa.upn.mx/hemeroteca/ world-mainmenu-26/107-num02/209-el-servicio-social-y-la-tutoriaen-la-upn-unidad-ajusco

Ortega, E. (2013). Eventos reflexivos a favor de la diversidad cultural en México: esbozo de una política intercultural del lenguaje desde la racionalidad sociolingüística (Tesis de doctorado inédita). Universidad Autónoma Metropolitana, UAM-Iztapalapa, México.

Pérez, C. (2012). Analfabetismo indígena, tres veces más alto que la media. Recuperado de http://www.educacionyculturaaz.com/noticias/ analfabetismo-indigena-tres-veces-mas-alto-que-la-media/

Rebolledo, N. (2005). Interculturalismo y autonomía. Las universidades indígenas y las políticas de alteridad. En C. Navarro (Coord.), La mala educación en tiempos de la derecha (pp. 112-135). México D.F.: Porrúa.

Rodríguez, P. y Rivera, T. (1996). La diversidad cultural y el entendimiento posible. En H. Muñoz y P. Lewin (Coord.), El significado de la diversidad lingüística y cultural (pp. 139-151). México D.F.: Universidad Autónoma Metropolitana, UAM-Iztapalapa e Instituto Nacional de Antropología e Historia -Oaxaca.

Romo, A. y Hernández, P. (2005). Evaluación del Programa de tutoría a estudiantes indígenas. México D.F.: Asociación Nacional de Universidades e Instituciones de Educación Superior, ANUIES. 
Ruiz, J. (1996). Metodología de la investigación cualitativa. Bilbao: Universidad de Deusto.

Saettele, H. (1978). Reflexividad del lenguaje e ideología lingüística. Arte, Sociedad e Ideología, 2, 27-36.

Salinas, G. y Avilés, M. (2003). Formación de docentes en y para la diversidad. En M. Bertely (Coord.), Educación, derechos sociales y equidad. Tomo I. Educación y diversidad cultural, educación y medio ambiente (pp. 165238). México D.F.: Consejo Mexicano de Investigación Educativa A.C, COMIE.

Schmelkes, S. (junio 2003). La política de la educación bilingüe intercultural en México. En I. Hernaiz, Educación en la diversidad: Experiencias y desafios desde la educación intercultural bilingüe. Seminario organizado por el Instituto Internacional de Planificación Educativa de la Unesco de Buenos Aires y la Coordinación General de Educación Intercultural Bilingüe de la Secretaría de Educación Pública de México, Ciudad de México.

Searle, J. (1991). Intentionality. An essay in the philosophy of mind. Cambridge: Cambridge, University Press.

Universidad Autónoma Benito Juárez, UABJO (2010). Datos estadísticos. Dirección de Servicios Escolares. Oaxaca: Autor.

Universidad Pedagógica Nacional, UPN (2011). Datos estadísticos de ingreso a la LEPEPMI'90 promoción 2010. Subdirección académica de la UPN unidad 201. Oaxaca: Autor.

Velasco, S. y Jablonska, A. (Coord.). (2010). Construcción de politicas educativas interculturales en México: debates, tendencias, problemas, desafíos. México D.F.: Universidad Pedagógica Nacional. 